\title{
Breaking waves: To foam or not to foam?
}

\author{
Petr Karnakov $\odot,{ }^{1}$ Sergey Litvinov $\odot,{ }^{1}$ Jean M. Favre $\odot,{ }^{2}$ and Petros Koumoutsakos $\odot{ }^{1, *}$ \\ ${ }^{1}$ Computational Science and Engineering Laboratory, ETH Zurich, Clausiusstrasse 33, \\ 8092 Zurich, Switzerland \\ ${ }^{2}$ Swiss National Supercomputing Centre, Via Trevano 131, 6900 Lugano, Switzerland
}

(Received 25 August 2020; published 12 November 2020)

\begin{abstract}
This paper is associated with a video winner of a 2019 American Physical Society's Division of Fluid Dynamics (DFD) Gallery of Fluid Motion Award for work presented at the DFD Gallery of Fluid Motion. The original video is available online at the Gallery of Fluid Motion, https://doi.org/10.1103/APS.DFD.2019.GFM.V0018.
\end{abstract}

DOI: 10.1103/PhysRevFluids.5.110503

Breaking waves entrain air bubbles that rise to the surface [1,2]. In clean fresh water, the bubbles disappear as they coalesce with each other or with the atmosphere. Yet in seawater, the bubbles cluster up on the surface and appear as foam. The reason is that surfactants and electrolytes [3-5] found in seawater prevent or delay coalescence as they stabilize the thin liquid films separating the bubbles. In this work, we show how prevention of coalescence changes the behavior of bubbles entrained by breaking waves. We compare two limiting cases, with and without coalescence, on a series of volume-of-fluid simulations of increasing complexity.

Conventional volume-of-fluid simulations correspond to the first case, bubbles always coalesce at a distance below one computational cell since the method is unable to describe multiple interfaces in the same cell. For coalescence prevention, we use an implementation [6] of the multimarker volume-of-fluid method [7,8] that removes its dependence on the number of bubbles and makes it applicable to systems with thousands of noncoalescing bubbles. The source code of our solver Aphros, documentation, and examples of simulations are available on GitHub [9] https://github. com/cselab/aphros.

The simplest setup is the forced collision of two bubbles shown in Fig. 1. The bubbles, initially separated, move towards each other under the force applied to the outer sides. Coalescence, if allowed, starts with a neck propagating along the surface of the newly formed bubble. Our simulations $[10,11]$ describe this process in close agreement with experimental data [12]. Without coalescence, the bubbles deform and eventually repulse.

Now we consider a column of bubbles generated by a periodic source, under the surface of water. With coalescence, each bubble rises towards the surface, and rapidly bursts creating radial capillary waves. Without coalescence, in Fig. 2, the bubbles cluster up on the surface. This clustering is driven by buoyancy since each bubble creates an elevation that attracts other bubbles. We obtain the same cluster of bubbles experimentally. In a tank of soapy water, we generate small bubbles of about $2 \mathrm{~mm}$ in diameter by injecting air at a fixed pressure through a thin tube.

\footnotetext{
*petros@ethz.ch
}

Published by the American Physical Society under the terms of the Creative Commons Attribution 4.0 International license. Further distribution of this work must maintain attribution to the author(s) and the published article's title, journal citation, and DOI. 

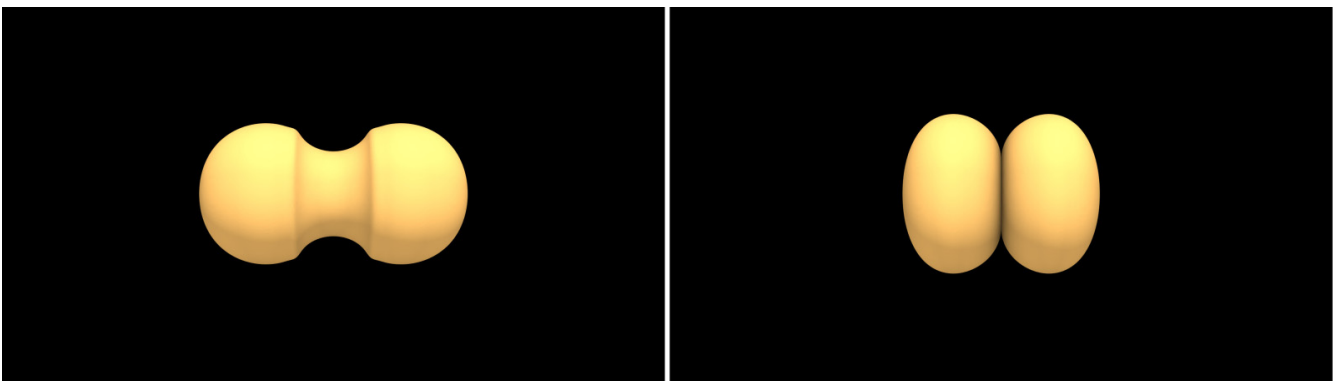

FIG. 1. Collision of two bubbles with coalescence (left) and without coalescence (right).
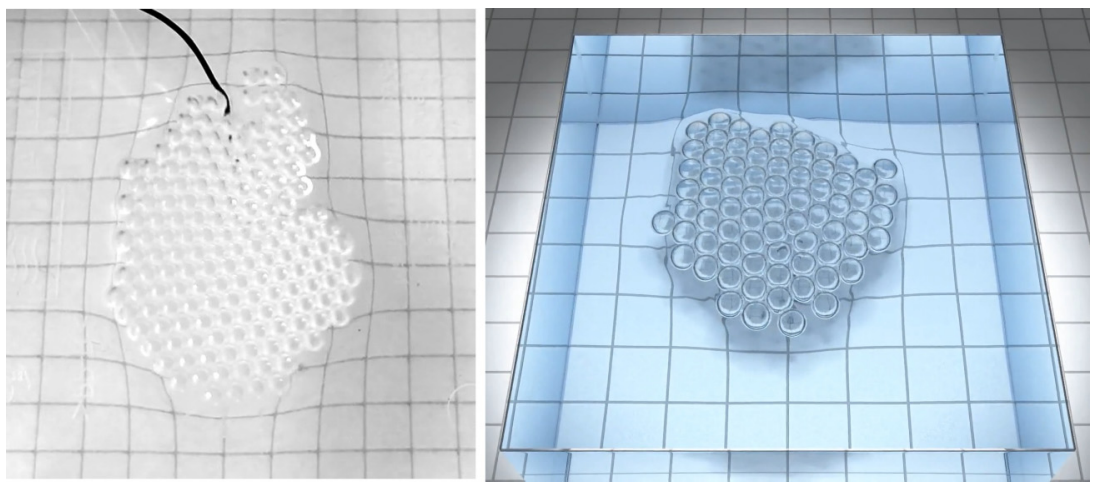

FIG. 2. Cluster of bubbles generated by a periodic source: experiment in water with surfactant (left) and simulation without coalescence (right). The square cells are $4 \mathrm{~mm}$ wide.
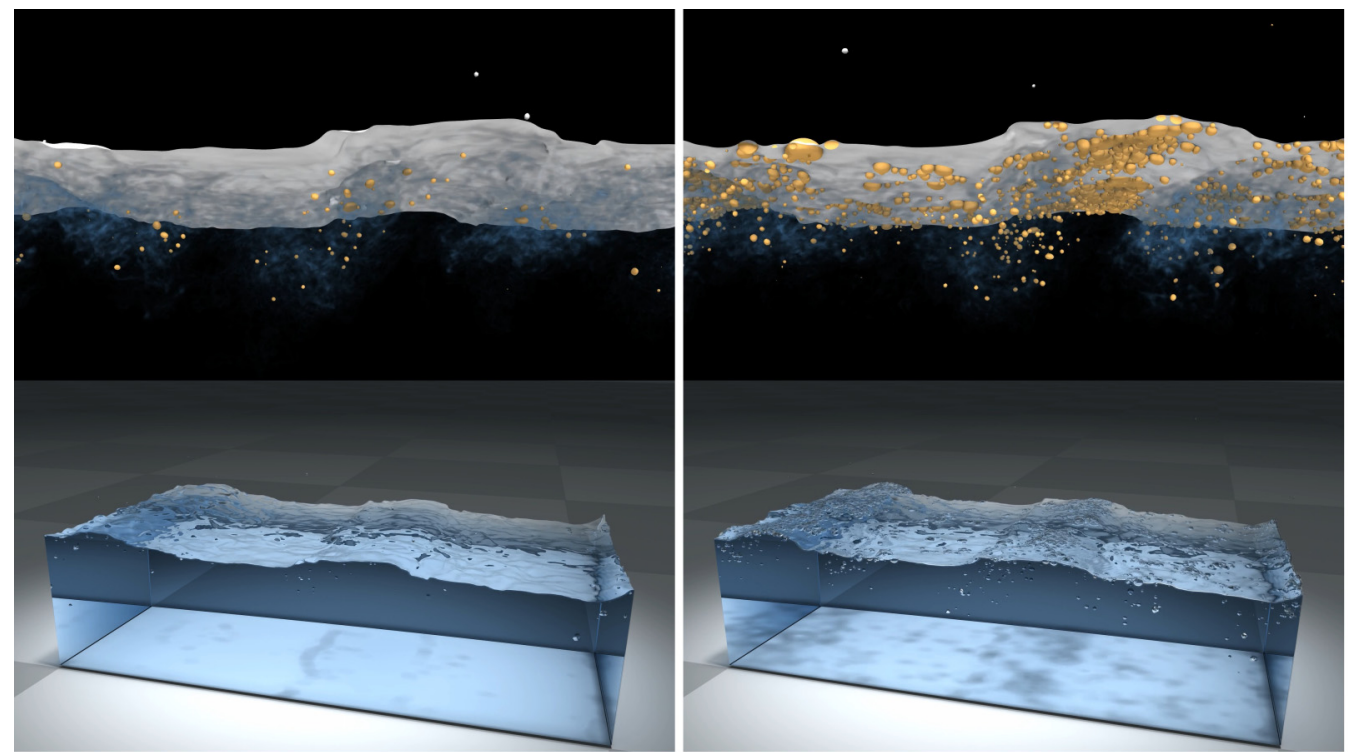

FIG. 3. Bubbles generated by breaking waves with coalescence (left) and without coalescence (right). Bubbles highlighted with orange (top) and rendering using path tracing (bottom). 
Finally, we consider a breaking wave using initial conditions [13] with a steepness $\varepsilon=0.65$ and a wavelength of $27 \mathrm{~cm}$. The wave captures a tube of air that is then divided into small bubbles. A snapshot is shown in Fig. 3. As expected, with coalescence the bubbles reach the surface and disappear. Prevention of coalescence makes the bubbles cluster up as foam.

We have illustrated the two limiting cases of coalescence prevention. However, depending on the flow conditions and the concentration of surfactants, bubbles can undergo partial coalescence. For example, foam on seawater decays over time. Future work will deal with such intermediate cases.

This research is funded by Grant No. CRSII5_173860 of the Swiss National Science Foundation. The authors acknowledge the use of computing resources from CSCS (projects s754 and s931).

[1] G. B. Deane and M. D. Stokes, Scale dependence of bubble creation mechanisms in breaking waves, Nature (London) 418, 839 (2002).

[2] K. T. Kiger and J. H. Duncan, Air-entrainment mechanisms in plunging jets and breaking waves, Annu. Rev. Fluid Mech. 44, 563 (2012).

[3] V. Craig, B. Ninham, and R. Pashley, Effect of electrolytes on bubble coalescence, Nature (London) 364, 317 (1993).

[4] L. A. Del Castillo, S. Ohnishi, and R. G. Horn, Inhibition of bubble coalescence: Effects of salt concentration and speed of approach, J. Colloid Interface Sci. 356, 316 (2011).

[5] K. Schilling and M. Zessner, Foam in the aquatic environment, Water Res. 45, 4355 (2011).

[6] P. Karnakov, F. Wermelinger, S. Litvinov, and P. Koumoutsakos, Aphros: High performance software for multiphase flows with large scale bubble and drop clusters, in Proceedings of the Platform for Advanced Scientific Computing Conference, Geneva, Switzerland (ACM Press, New York, NY, 2020), pp. 1-10.

[7] E. Coyajee and B. J. Boersma, Numerical simulation of drop impact on a liquid-liquid interface with a multiple marker front-capturing method, J. Comput. Phys. 228, 4444 (2009).

[8] N. Balcázar, O. Lehmkuhl, J. Rigola, and A. Oliva, A multiple marker level-set method for simulation of deformable fluid particles, Int. J. Multiphase Flow 74, 125 (2015).

[9] Aphros: Parallel solver for incompressible multiphase flows, https://github.com/cselab/aphros (2020).

[10] P. Karnakov, S. Litvinov, and P. Koumoutsakos, A hybrid particle volume-of-fluid method for curvature estimation in multiphase flows, Int. J. Multiphase Flow 125, 103209 (2020).

[11] S. M. H. Hashemi, P. Karnakov, P. Hadikhani, E. Chinello, S. Litvinov, C. Moser, P. Koumoutsakos, and D. Psaltis, A versatile and membrane-less electrochemical reactor for the electrolysis of water and brine, Energy Environ. Sci. 12, 1592 (2019).

[12] Á. M. Soto, T. Maddalena, A. Fraters, D. Van Der Meer, and D. Lohse, Coalescence of diffusively growing gas bubbles, J. Fluid Mech. 846, 143 (2018).

[13] A. Iafrati, Numerical study of the effects of the breaking intensity on wave breaking flows, J. Fluid Mech. 622, 371 (2009). 